\title{
Efeito agudo da estimulação elétrica nervosa transcutânea (TENS) na lombalgia
}

\section{Acute effect of transcutaneous nerve electric stimulation (TENS) on lombalgy}

(D) Bruna de Lima Rosa ${ }^{1}$, Bruna Albani Borba ${ }^{1}$, (DTaiana Boeira Oliveira ${ }^{1}$, (Dilena Lumertz ${ }^{1}$, (D)Josué Nascente dos Santos ${ }^{1}$, @Marcelo Baptista Dohnert ${ }^{2}$, (Dodrigo Boff Daitx ${ }^{1}$

\section{RESUMO}

Resumo A lombalgia é um problema extremamente comum, que afeta mais pessoas do que qualquer outra afecção. Acarreta em um quadro de dor, perda de função e redução da qualidade de vida. Isto posto, o tratamento conservador é de suma importância para a reabilitação de pacientes com lombalgia. Objetivo: Avaliar o efeito agudo da estimulação elétrica transcutânea (TENS) em portadores de lombalgia. Métodos: Série de casos, no qual participaram 66 pacientes. Foi realizado uma única intervenção com TENS no modo acupuntural, frequência de $70 \mathrm{~Hz}$, tempo de pulso de 150 us, com duração de 40 minutos. Foram avaliados o nível de dor, flexibilidade de isquiotibiais, força muscular e estabilização do complexo lombopélvico pré, imediatamente após e 24 horas após a intervenção. Resultados: 0 nível de dor, flexibilidade de isquiotibiais e força muscular dos extensores de tronco apresentaram melhora imediatamente após intervenção $(p<0,05)$. Após 24 horas, houve aumento significativo da dor em comparação ao pós intervenção $(p<0,05)$, porém ainda se verificou um efeito analgésico e de melhora da flexibilidade em comparação a avaliação pré. A força muscular melhorou de forma significativa pós intervenção e manteve-se 24 horas após $(p<0,05)$. Os testes de estabilidade apresentaram maior tempo de manutenção da postura imediatamente após a aplicação da TENS, porém com redução deste feito pós 24 horas $(p<0,05)$. Conclusão: A TENS demonstrou redução do quadro álgico, melhora da força de tronco, flexibilidade de isquiotibiais e da estabilização lombopélvica imediatamente após a intervenção. Estes efeitos se mantiveram 24 horas após, porém não com a mesma intensidade.

Palavras-chave: Terapia por Estimulação Elétrica, Dor Lombar, Modalidades de Fisioterapia, Reabilitação

\footnotetext{
${ }^{1}$ Universidade Luterana do Brasil - ULBRA

${ }^{2}$ Universidade de Gurupi - UnirG
}

\section{Correspondência}

Rodrigo Boff Daitx

E-mail: rodrigo.roseta@hotmail.com

Recebido: 05 Janeiro 2020

Aceito: 27 Junho 2020

\section{Como citar}

Rosa BL, Borba BA, Oliveira TB, Lumertz M, Santos $J N$, Dohnert MB, et al. Efeito agudo da estimulação elétrica nervosa transcutânea (TENS) na lombalgia. Acta Fisiatr. 2020;27(1):34-40.

DOI: 10.11606/issn.2317-0190.v27i1a171150

\begin{abstract}
Low back pain is an extremely common problem that affects more people than any other condition. It causes pain, loss of function and reduced quality of life. That said, conservative treatment is of paramount importance for the rehabilitation of patients with low back pain. Objective: To evaluate the acute effect of transcutaneous electrical stimulation (TENS) in patients with low back pain. Methods: Case series, in which 66 patients participated. A single intervention with TENS was performed in acupuncture mode, frequency of $70 \mathrm{~Hz}$, pulse time of $150 \mathrm{us}$, lasting 40 minutes. The level of pain, hamstring flexibility, muscle strength and stabilization of the lumbopelvic complex pre, immediately after and 24 hours after the intervention were evaluated. Results: The level of pain, hamstring flexibility and muscle strength of the trunk extensors improved immediately after intervention ( $p<0.05$ ). After 24 hours, there was a significant increase in pain compared to the post-intervention period ( $p<0.05$ ), but there was still an analgesic effect and improved flexibility compared to the pre evaluation. Muscle strength improved significantly after the intervention and was maintained 24 hours later $(p<0.05)$. The stability tests showed a longer posture maintenance time immediately after the application of TENS, but with a reduction of this done after 24 hours $(p<0.05)$. Conclusion: TENS demonstrated a reduction in pain, improvement in trunk strength, hamstring flexibility and lumbopelvic stabilization immediately after the intervention. These effects were maintained 24 hours later, but not to the same degree.
\end{abstract}

Keywords: Electric Stimulation Therapy, Low Back Pain, Rehabilitation 


\section{INTRODUÇÃO}

As alterações que geram a degeneração discal são consideradas causas multifatoriais identificadas por modificações celulares e na bioquímica do disco que resultam em defeitos na sua estrutura. ${ }^{1}$ A consequência deste processo é o surgimento da protrusão discal (PD) propiciando o aumento da pressão discal e subsequente probabilidade de hérnia, gerando dor e demais sintomas característicos dos nervos periféricos em compressão. ${ }^{1} \mathrm{~A}$ lombalgia com presença de PD ocorre quando há extensão assimétrica ou focal do disco para além do espaço intermédio. ${ }^{2}$ Neste caso, a base ou origem do material do disco tem um diâmetro maior do que qualquer outra dimensão da protrusão. ${ }^{2}$

O tratamento conservador é escolhido na maioria dos casos e tem como objetivos aliviar a dor, aumentar a capacidade funcional e diminuir a progressão da doença. ${ }^{3}$ Várias estratégias de tratamento vem sendo utilizadas como programas de exercícios com controle motor dos músculos do tronco através de exercícios de estabilização do Core, exercícios de fortalecimento e alongamentos. ${ }^{4} \mathrm{Na}$ prática fisioterapêutica, diferentes agentes eletrofísicos são utilizados para obter alívio da dor relacionado aos problemas musculoesqueléticos, a fim de complementar outras intervenções terapêuticas, particularmente a realização de exercícios corretivos. ${ }^{5} \mathrm{Em}$ indivíduos com dor lombar, deuma forma geral, o alívio imediato da dor aguda durante as primeiras sessões de fisioterapia fornece o terreno adequado para o início dos exercícios. ${ }^{5}$

Assim, escolher a modalidade adequada para atingir esse objetivo é uma questão importante na prática clínica. ${ }^{5}$ A TENS é uma técnica amplamente utilizada dentro da eletroterapia, sendo considerada um tratamento coadjuvante simples e seguro, podendo ser efetiva para manejo da dor lombar de diversas causas, mecânicas ou radiculares. ${ }^{4}$

A estimulação elétrica nervosa transcutânea (TENS) é uma das modalidades mais comumente usadas, com evidência moderada a forte de sua capacidade de modular a dor em diferentes condições de dor. ${ }^{5}$ Foi demonstrado que a TENS pode diminuir a dor durante, logo após e por um intervalo de tempo determinado após aplicação nos tecidos moles lesionados 5 . É o recurso não farmacológico mais utilizado na prática clinica fisioterapêutica. ${ }^{6}$ Caracteriza-se como uma corrente de baixa intensidade que produz impulsos elétricos com frequência entre zero e $200 \mathrm{~Hz}$, eficaz no tratamento álgico de desordens musculoesqueléticas. ${ }^{6}$ Os impulsos da TENS estimulam as fibras mielinizadas do tipo $A$, que excitam os interneurônios do corno posterior da medula espinhal e inibem os impulsos nociceptivos das fibras A-delta e C, mecanismo este conhecido como Teoria das Comportas. $^{7}$

\section{OBJETIVO}

O presente estudo teve por objetivo avaliar os efeitos agudos da TENS em pacientes com lombalgia.

\section{MÉTODOS}

Trata-se de uma série de casos com 66 indivíduos portadores de lombalgia. $O$ estudo foi realizado na clínica escola de fisioterapia da Ulbra, campus de Torres-RS, no período de janeiro a outubro de 2018. O estudo foi aprovado pelo Comitê de Ética e Pesquisa da Universidade Luterana do Brasil sob parecer no 2.306 .760 .

Foram incluídos pacientes de ambos os gêneros, com idade entre 18 e 70 anos que apresentavam lombalgia decorrente de PD confirmada através de ressonância magnética ou tomografia computadorizada e que não estavam realizando nenhum outro tipo de abordagem fisioterapêutica ou tratamento medicamentosos para dor lombar no momento do estudo e que assinaram o Termo de Consentimento Livre e Esclarecido (TCLE) previamente ao ingresso no estudo.

Foram excluídos do estudo participantes que apresentavam patologias graves da coluna vertebral (fratura, tumores e patologias inflamatórias, tais como espondilite anquilosante), com cirurgia prévia na região lombar e colocação de implante metálico, pacientes com doença cardiovascular e cardiorrespiratória grave, mulheres grávidas, utilização de marcapasso cardíaco, dor de origem desconhecida, lesões cutâneas no local de aplicação da TENS, participantes com algum distúrbio neurológico e que não conseguiram realizar os testes propostos e os que utilizavam algum tratamento medicamento analgésico.

Após preencherem os critérios de elegibilidade, os pacientes do estudo receberam informações sobre a pesquisa e foram orientados a assinar o TCLE de acordo com a Resolução do Conselho Nacional da Saúde. O protocolo de avaliação foi realizado por um avaliador independente em três momentos do estudo: previamente a intervenção, imediatamente após o término da intervenção e 24 horas após a intervenção.

Inicialmente foi realizada a coleta dos dados demográficos do paciente. Posteriormente, foram coletados os dados antropométricos como o peso e estatura. Após, foi realizada a avaliação do nível de dor através da Escala Visual Analógica (EVA). A flexibilidade da parte posterior de tronco e membros inferiores foi realizada através do banco de Wells. A força muscular dos músculos extensores do troncofoi avaliada através do dinamômetro dorsal.

A estabilidade e coordenação lombopélvica foi avaliada através dos testes de estabilidade lombopélvica, sendo eles o Single leg squat (agachamento unilateral), Runner pose test (Teste do corredor), teste de instabilidade em prono, teste de força em ponte lateral realizado unilateralmente pelo lado dominante do paciente e o Teste de Sorenson (teste de resistência extensora).

O protocolo de aplicação da TENS ocorreu através de uma única intervenção com duração de 40 minutos. Foi utilizado um aparelho da marca TENSMED IV Digital Four Channel TENS unit 4034. Os parâmetros selecionados foram da TENS no modo acupuntural, com dois canais e quatro eletrodos, com uma frequência de $70 \mathrm{~Hz}$, tempo de pulso de 150 us e a intensidade foi ajustada para o nível de conforto do paciente, mas suficiente para gerar contrações musculares visíveis. ${ }^{8,9}$

O paciente foi posicionado em decúbito ventral sobre a maca e os eletrodos foram posicionados de forma cruzada, aplicados sobre a musculatura paravertebral bilateral de L3 a S1. ${ }^{9}$ A aplicação do protocolo de intervenção foi realizada por pesquisadores previamente treinados e familiarizados com o protocolo.

Foi utilizado como desfecho primário do estudo o índice de dor avaliado através da escala analógica visual de dor (EVA) e, baseado no estudo realizado por Rajfur et al. ${ }^{10}$ estimamos a média e desvio padrão do escore de dor dos participantes do estudo de $7,72 \pm 1,35$ no grupo TENS e 7,34 $\pm 1,57$ no grupo controle; uma média e desvio padrão da pontuação do escore EVA final de 2,11 \pm 0,34 no grupo TENS e de $4,11 \pm 0,69$ para o grupo controle após o tratamento, utilizando um poder de força do estudo de $80 \%$ e um nível de significância de $95 \%$, chegou-se ao número estimado de 66 sujeitos no grupo de intervenção. Acreditando que as perdas e recusas chegariam em torno de $20 \%$, chegamos ao número final de 53 sujeitos. 
Foi utilizado o Statistical Package for the Social Sciences (SPSS) versão 17.0 como banco de dados e pacote estatístico. Os dados foram duplamente digitados a fim de evitar erros de digitação e expressos em média e desvio padrão. Após, foram analisados estatisticamente pelo teste paramétrico Análise de Variância (ANOVA) para medidas repetidas para análise.

Para as variáveis categóricas foi utilizado o teste de Qui-quadrado. Para as variáveis não paramétricas foram utilizados os testes de Kruskal-Wallis e Mann-Whitney, respectivamente. $O$ nível de significância estabelecido para o teste estatístico foi de $p<0,05$.

\section{RESULTADOS}

Foram inicialmente selecionados e avaliados 78 participantes. Destes, 12 foram excluídos sendo quatro por não apresentarem nível significativo de dor, um por utilizar marcapasso cardíaco, dois por apresentar prótese metaliza na coluna lombar, uma portadora de espondilolistese, um por não conseguiu realizar os testes e três por nãocomparecer na reavaliação após 24 horas. Portanto, a amostra contou com 66 participantes incluídos no estudo. As características gerais da amostra estão descritas na Tabela 1.

O nível de dor reduziu de forma significativa imediatamente após a intervenção, permanecendo esta analgesia após 24 horas da intervenção. Porém, ocorreu um aumento da dor 24 horas após a aplicação do TENS em comparação a avaliação pós intervenção. Na avaliação inicial, o nível de dor foi de $6,68 \pm 0,22$ pontos, reduzindo para 2,19 $\pm 0,28$ pontos no pós intervenção imediato $(p=0,000)$. Após 24 horas, o nível de dor elevou para $4,18 \pm 0,28$ pontos, sendo ainda inferior a dor inicial $(p=0,000)$, porém superior a dor imediatamente após a intervenção $(p=0,001)$ (Figura 1).

A flexibilidade dos isquiotibiais, avaliada através do banco de Wells, aumentou de 20,41 $\pm 9,9 \mathrm{~cm}$ na avaliação inicial para $23,78 \pm 10,2$ $\mathrm{cm}$ na avaliação pós intervenção $(p=0,000)$. Vinte e quatro horas após a intervenção, a flexibilidade reduziu para 21,74 $\pm 10,03 \mathrm{~cm}$, porém ainda significativamente maior que a verificada na avaliação inicial $(p=0,05)$

A força muscular de tronco apresentou um aumento estatisticamente significativo na avalição pós intervenção, comparado com a avaliação inicial, e se manteve 24 horas após a aplicação da TENS. A força inicial foi de 43,6
Tabela 1. Características demográficas da amostra do estudo $(n=66)$

\begin{tabular}{lcc}
\hline Variável & $\mathbf{N}$ & $\%$ \\
\hline Sexo & 18 & 27,3 \\
Masculino & 48 & 72,7 \\
Feminino & $48,89 \pm 9,96$ & \\
\hline Idade, anos (dp) & & \\
Ocupação & 14 & 21,2 \\
Do lar & 5 & 15,0 \\
Aposentado(a) & 37 & 7,5 \\
Serviços Gerais & $8,47 \pm 6,74$ & \\
Outros & $77,73 \pm 15,58$ & \\
\hline Tempo de dor, & $165,53 \pm 8,69$ \\
anos & $28,24 \pm 4,25$ \\
\hline Peso, kg (dp) & \\
\hline Estatura, cm (dp) & \\
\hline IMC, kg/cm² (dp) & \\
\hline
\end{tabular}

$\mathrm{N}$ : número de participantes; \%: porcentagem; dp: desvio padrão; $\mathrm{kg}$ : quilograma; cm: centímetros; IMC: índice de massa corporal; $\mathrm{Kg} / \mathrm{cm} 2$ : quilogramas por centímetro quadrado.

$\pm 23,46 \mathrm{~kg}$, aumentando para 48,84 $\pm 24,3$ $\mathrm{kg}$ na avaliação pós TENS ( $\mathrm{p}=0,006)$. Após 24 horas, a força se manteve em 47,65 $\pm 25,72$ $\mathrm{kg}(\mathrm{p}=0,03$ em comparação a avaliação inicial) (Figura 2).

Os testes de estabilidade lombopélvica Single Leg Squat test, Runner Pose test e o teste de instabilidade em prono demonstraram uma melhora significativa tanto imediatamente pós TENS quanto na avaliação 24 horas Pós TENS (Tabela 2).

subindo para 20,95 $\pm 1,86$ segundos pós TENS $(p=0,002)$ e, 24 horas pós TENS, para $21,04 \pm 1,9$ segundos $(p=0,002)$.

$\mathrm{O}$ teste de Sorenson apresentou um aumento no tempo de manutenção da postura apenas na avalição pós TENS $(p=0,05)$, passando de 18,77 $\pm 14,2$ segundos na avaliação inicial para $22,21 \pm 14,84$ segundos pós TENS. Vinte e quatro horas pós TENS, o tempo reduziu para $20,74 \pm 14,89$ segundos (Figura 3).

\section{DISCUSSÃO}

No presente estudo, investigamos o efeito imediato da aplicação da TENS sobre a lombalgia com presença de achado imagenológico de PD, bem como o tempo de duração deste possível efeito analgésico. A lombalgia decorrente da degeneração do disco intervertebral lombar é uma das doenças degenerativas da coluna vertebral mais comuns que podem levar a dor lombar e radicular irradiada para os membros inferiores. ${ }^{11}$ Essa condição gera incapacidade motora de vários graus para o desenvolvimento de diversas atividades e leva a um comprometimento relevante da qualidade de vida desses individuos. ${ }^{12}$

Observou-se, no presente estudo, um número maior de participantes do gênero feminino, o que é facilmente justificável, pois as mulheres apresentam uma maior prevalência de dor crônica e também uma maior percepção quanto aos sintomas e sinais das doenças. ${ }^{13,14}$ Além disso, contam com fatores importantes como uma exposição maior aos trabalhos repetitivos e maior intensidade dos afazeres domésticos, apresentando ainda grande velocidade de trabalho em posições não ergonômicas. ${ }^{13,15} \mathrm{As}$ mulheres também apresentam características anatômicas diferentes como menor estatura, menor massa muscular, menor massa óssea, articulações mais frágeis e que, ao esforço físico extenuante, encontram-se menos adaptadas, podendo acarretar mais sobrecarga na coluna. ${ }^{13,15}$

A amostra deste estudo apresentou um índice de massa corporal (IMC) elevado, o que é plausível, visto que a dor crônica na coluna está associada ao sobrepeso e a obesidade. ${ }^{13}$ A fraqueza muscular, o desgaste dos discos vertebrais e o surgimento dos processos inflamatórios são consequências da sobrecarga gerada com o aumento do peso corporal, favorecendo o aparecimento de lombalgia e hérnia de disco, entre outras doenças na coluna. ${ }^{13}$ A perda de peso, quando priorizada, pode diminuir a dor e a incapacidade. ${ }^{13}$ Azevedo et al. ${ }^{16}$ investigaram a relação entre lombalgia e sobrepeso através do IMC, adipometria abdominal e queixa de dor lombar. ${ }^{16}$ Os resultados demonstraram que os indivíduos que apresentaram maior índice de massa corpórea (53\%) denotaram maior prevalência de lombalgia, quando comparados aos de peso normal (47\%). ${ }^{16}$

Verificou-se, neste estudo, uma redução expressiva do nível de dor imediatamente após a intervenção. Porém, 24 horas após a aplicação, mesmo ainda se verificando analgesia, ocorreu um aumento expressivo do nível de dor. Schulz et al. ${ }^{6}$ relataram que a eletroestimulação de baixa frequência oportuniza contração muscular e, assim, gera ativação dos mecanismos de liberação dos opioides endógenos, aumentando temporariamente o fluxo sanguíneo arterial no local de aplicação, propiciando quadro de analgesia maior, sendo efetivo na modulação da dor clínica..$^{6,13}$ 


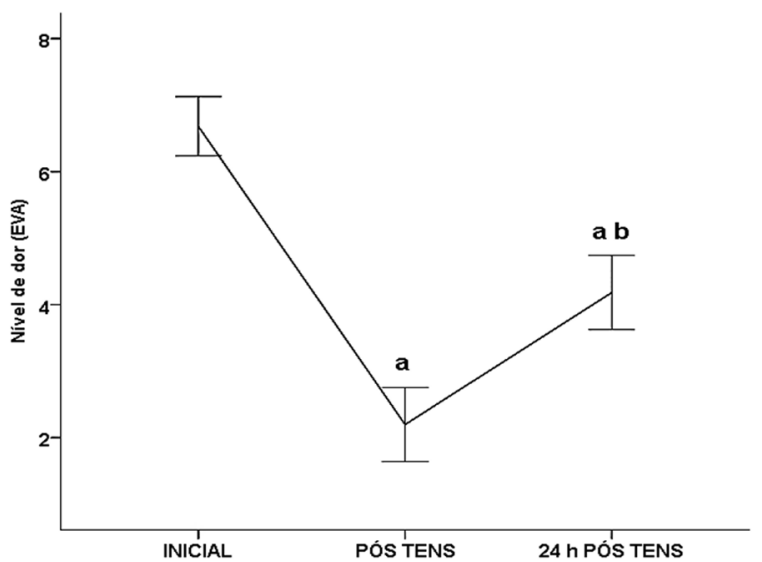

$a=p<0,05$ em relação a avaliação inicial; $b=p<0,05$ em relação a avaliação pós TENS; ANOVA para medidas repetidas

Figura 1. Avaliação do nível de dor Inicial, Pós TENS e 24h Pós TENS através da EVA

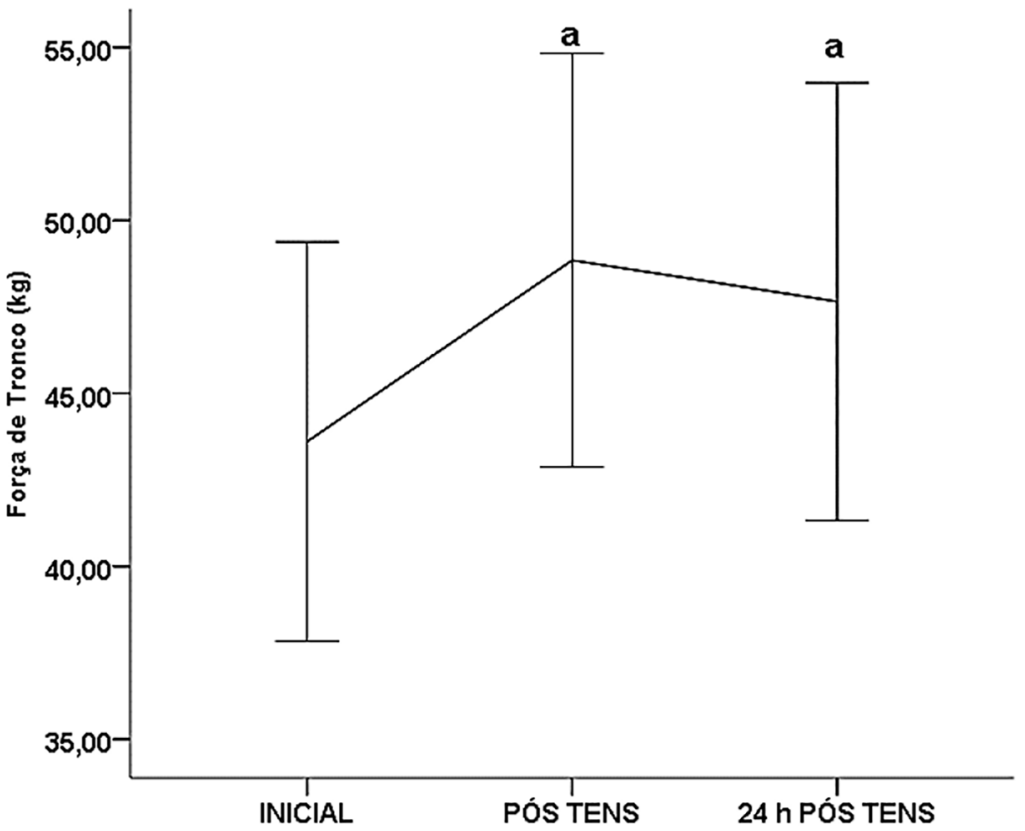

$a=p<0,05$ em relação a avaliação inicial; ANOVA para medidas repetidas

Figura 2. Avaliação da força dinamométrica de tronco Inicial, Pós TENS e 24h Pós TENS, mensurada através do dinamômetro dorsal

Diante disso, a TENS no modo acupuntural tem uma particularidade no efeito pós tratamento, devido à liberação progressiva e lenta destes opióides pelo sistema nervoso central, mantendo a analgesia no local por um período após a aplicação. ${ }^{6}$ No entanto, permanece em média, apenas cerca de seis horas. ${ }^{6}$ Porém, Johnson et al. ${ }^{17}$ relataram que a experiência clínica sugere que a TENS pode aliviar qualquer tipo de dor crônica, mas as revisões sistemáticas são inconclusivas para dor lombar. ${ }^{17}$ Os autores ainda expuseram que algumas revisões citam que o alívio da dor dura somente enquanto o estimulo estiver sendo aplicado, admitindo-se que ocorra uma considerável acomodação. ${ }^{17}$ Desta forma, supõe-se que os pacientes podem precisar administrar TENS ao longo do dia para obter os melhores efeitos e por um período mais prolongado. ${ }^{17}$

Considerando os efeitos agudos, Tugay et al. ${ }^{18}$ encontraram efeitos estatisticamente superiores da corrente interferencial em relação à dor lombar imediatamente após a estimulação elétrica e oito horas após a sua aplicação, mas essa diferença desapareceu 24 horas após a aplicação do estímulo elétrico. Essa analgesia produzida pela estimulação elétrica pode ser interpretada como um aumento no limiar da dor e a TENS convencional pode ser responsável por esse efeito, uma vez que interfere na transmissão das sensações dolorosas para os níveis supraespinhais. ${ }^{19}$ Tanto a TENS como a corrente interferencial com baixa frequência de estímulo e alta intensidade podem atingir analgesia via liberação de opióides endogenos. ${ }^{19}$

No estudo de Ebadi et al. ${ }^{20} 30$ pacientes com lombalgia receberam abordagem em uma única sessão divididos em grupo corrente diadinâmica e grupo TENS. ${ }^{20} \mathrm{~A}$ intervenção de estimulação elétrica teve duração de 10 minutos no grupo corrente diadinâmica e 15 minutos no grupo TENS, com frequência de $120 \mathrm{~Hz}$ e tempo de pulso equivalente a 120 pps. ${ }^{20} \mathrm{~A}$ avaliação foi realizada pré intervenção e pós intervenção, 20 minutos depois da aplicação e após 48 horas da intervenção. ${ }^{20}$

Os resultados demonstraram uma diminuição significativa da dor imediatamente após a intervenção e 20 minutos após no grupo TENS. ${ }^{20}$ Já o limiar de dor por pressão aumentou imediatamente após a aplicação em ambos os grupos, mas não se manteve nas avaliações posteriores..$^{20}$ Estes resultados corroboram com o presente estudo, no qual também houve diminuição imediata dos níveis de dor no paciente logo após aplicação da TENS. Considerando que a frequência pode não determinar a eficácia da TENS, as evidências atuais mostram que a intensidade pode ser o fator chave para alcançar a redução ideal da dor. ${ }^{14}$

Mammari et al. ${ }^{21}$ realizaram um estudo prospectivo randomizado comparando dois grupos de pacientes com lombalgia, sendo 95 participantes tratados pela TENS e 66 tratados com infravermelho e ultrassom. ${ }^{21} \mathrm{O}$ tratamento foi de 12 semanas, sendo três sessões por semana para cada paciente. $O$ follow up foi de 12 meses. ${ }^{21} \mathrm{O}$ equipamento TENS foi programado na frequência de 20 $\mathrm{Hz}$ e tempo de pulso de $330 \mathrm{pps}$, com dois canais e quatro eletrodos. ${ }^{21}$ Os resultados demonstraram que o alívio da dor a curto e longo prazo foi significativamente melhor no 
Tabela 2. Resultados dos testes de estabilidade lombopélvica na avaliação Inicial, pós TENS e $24 h$ pós TENS $(n=66)$

Inicial Pós TENS 24 hs pós TENS

\begin{tabular}{|c|c|c|c|c|c|}
\hline \multicolumn{6}{|c|}{ Single Leg Squat test, n (\%) } \\
\hline Positivo & $51(77,3)$ & $18(27,3)$ & \multirow{2}{*}{ a } & $24(36,4)$ & \multirow{2}{*}{$a, b$} \\
\hline Negativo & $15(22,7)$ & $48(72,7)$ & & $42(53,6)$ & \\
\hline \multicolumn{6}{|c|}{ Runner Pose test, n (\%) } \\
\hline Positivo & $51(77,3)$ & $12(18,2)$ & \multirow{2}{*}{$a$} & $16(24,2)$ & \multirow{2}{*}{$a, b$} \\
\hline Negativo & $15(22,7)$ & $54(81,8)$ & & $50(75,8)$ & \\
\hline \multicolumn{6}{|c|}{$\begin{array}{l}\text { Teste de Instabilidade em } \\
\text { Prono, } n(\%)\end{array}$} \\
\hline Positivo & $29(43,9)$ & $3(4,5)$ & \multirow{2}{*}{$a$} & $22(33,3)$ & \multirow{2}{*}{$a, b$} \\
\hline Negativo & $37(56,1)$ & $63(95,5)$ & & $44(66,7)$ & \\
\hline
\end{tabular}

Seg = segundos; $d p=$ desvio padrão; $a=p<0,05$ em comparação com a avaliação inicial; $b=p<0,05$ em comparação com a avaliação pós TENS; Qui quadrado.

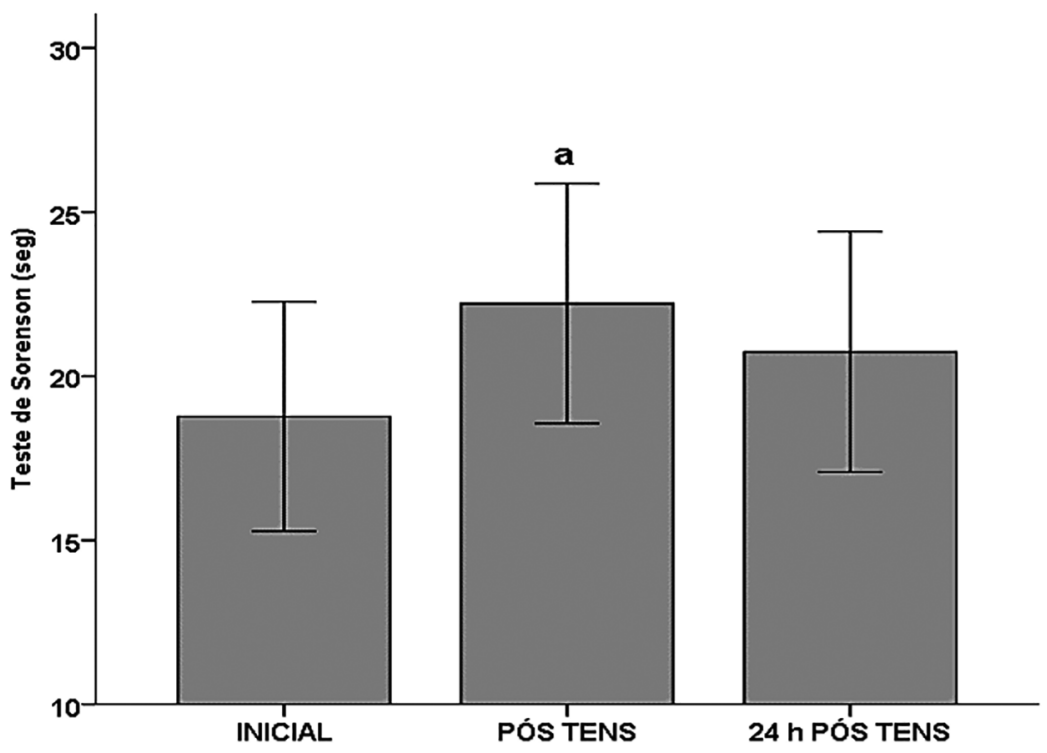

a p $<0,05$ em comparação ao pré intervenção; ANOVA para Medidas Repetidas

Figura 3. Resultados do Teste de Sorenson

grupo TENS, em comparação com o grupo que utilizou infravermelho e ultrassom. ${ }^{21} \mathrm{~A}$ longo prazo, a TENS também demonstrou resultados mais promissores em comparação ao outro grupo, reduzindo significativamente a sintomatologia e melhorando a qualidade de vida em pacientes com dor lombar crônica. ${ }^{21}$

Francisco et al. ${ }^{22}$ buscaram mensurar o efeito agudo da Facilitação Neuromuscular Proprioceptiva (PNF) associado à TENS e crioterapia na flexibilidade dos músculos isquiotibiais de mulheres saudáveis. ${ }^{22} \mathrm{~A}$ amostra foi composta por 60 voluntárias, distribuídas em grupo controle, grupo alongamento PNF isolado, grupo PNF associado a TENS e grupo PNF associado a crioterapia. ${ }^{22}$ Os resultados demonstraram que houve um aumento da flexibilidade comparando o pré e pós intervenção em todos os grupos, exceto no grupo controle. ${ }^{22} \mathrm{Na}$ análise intergrupos, observou-se diferença estatística apenas na comparação dos três grupos experimentais com o grupo controle. ${ }^{22}$

Os resultados demonstraram que 0 alongamento com PNF aplicado isoladamente ou associado à TENS e crioterapia é igualmente efetivo para aumentar a flexibilidade aguda dos músculos isquiotibiais. ${ }^{22}$ Basicamente dois mecanismos são referidos como responsáveis pelo aumento da amplitude de movimento (ADM) após o alongamento muscular. O primeiro fala na modificação na sensibilidade dos receptores de dor como fator responsável pelo aumento da tolerância ao alongamento e, consequentemente, pela efetividade das técnicas. ${ }^{22} \mathrm{O}$ segundo relata que alterações nas propriedades viscoelásticas dos tecidos, como decréscimo na tensão passiva da unidade músculotendínea imediatamente após o alongamento, são os principais responsáveis pelo ganho de flexibilidade. ${ }^{22}$

Diante destes resultados, em que houve uma maior flexibilidade pós TENS, e se prolongou até às $24 \mathrm{~h}$, ainda que com valores inferiores, porém maiores que da avaliação inicial, podemos sugerir que, com a analgesia, possivelmente ocorra um aumento da tolerância do indivíduo à manobra promovendo, assim, maior ganho de ADM.

No presente estudo a força dos extensores de tronco teve um aumento estatisticamente significativo na avalição pós intervenção comparada com a avaliação inicial. Após 24 horas, a força se manteve sugerindo que, ao proporcionar o bloqueio da dor advinda da discopatia degenerativa da coluna lombar, foi possível verificar um aumento estatisticamente significativo da força muscular de tronco.

Law et al. ${ }^{23}$ investigaram se a TENS isolada pode possibilitar a melhora da funcionalidade com relação a ADM do joelho. ${ }^{23}$ Os participantes foram designados em dois grupos de forma aleatória, um recebendo TENS e outro TENS placebo. ${ }^{23}$ Os parâmetros utilizados foram frequência equivalente a 100 $\mathrm{Hz}$, tempo de pulso de 200 pps e tempo de aplicação de 40 minutos. ${ }^{23}$

Os resultados demonstraram que o grupo TENS apresentou um aumento estatisticamente significativo na ADM máxima do joelho comparado com o grupo placebo. ${ }^{23}$ É possível que a TENS reduza a dor e o edema sobre a articulação, reduzindo a resistência ao movimento. ${ }^{23}$ A maioria das pessoas acreditam que existe um círculo vicioso entre dor e movimento. ${ }^{23} \mathrm{~A}$ dor é um fator importante que restringe o movimento. ${ }^{23}$ Teoricamente, quando a dor diminui, o espasmo muscular diminui e a mobilidade articular pode aumentar. ${ }^{23}$

Bertor et al. ${ }^{24}$ verificaram o efeito isolado e associado da classificação clínica da dor lombar e do nível de incapacidade sobre a capacidade funcional adquirida através da distância no teste de caminhada de seis minutos e 
utilizando o dinamômetro dorsal sobre a força dos músculos dorsais e membros inferiores. ${ }^{24}$ Foram avaliados 31 sujeitos divididos em três grupos: controle (sem distúrbios sistêmicos ou musculoesqueléticos), lombar específico e inespecifico. ${ }^{24}$ Os resultados demostraram que a lombalgia crônica influenciou na força muscular dorsal e dos membros inferiores (MMII) demostrando que o quadro álgico pode interferir e influenciar na força muscular de extensores de tronco. ${ }^{24}$

Indivíduos com dor lombar parecem diminuir a capacidade de controle da musculatura profunda local. A instabilidade é resultado de uma lesão tecidual que torna o segmento mais fraco ou insuficientemente resistente. No presente estudo, todos os testes de estabilidade lombopélvica apresentaram resultados positivos após a aplicação da TENS. Evidências demonstram que as TENS de alta e baixa frequência produzem efeitos analgésicos por diferentes mecanismos neurobiológicos. ${ }^{25}$

De acordo com a teoria da compota de dor, a TENS de alta frequência e baixa intensidade ativa as fibras $A \beta$ de grande diâmetro, que no nível da corno dorsal da medula inibem o imput nociceptivo recebido e transmitida por fibras $A \delta$ e $C$ de pequeno diâmetro e de condução lenta que inervam, espacialmente, áreas adjacentes da pele. ${ }^{25}$ Observa-se que a TENS de alta frequência e baixa intensidade produz um claro efeito analgésico à estimulação nociceptiva homotópica, ou seja, no mesmo segmento ou local. ${ }^{25}$

Entretanto, o efeito analgésico da TENS de alta frequência e baixa intensidade não pode ser totalmente explicado por um mecanismo de inibição homotópica, pois uma contribuição concomitante de um mecanismo de inibição descendente supraespinhal permanece uma possibilidade, conforme sugerido por vários achados em animais. ${ }^{25}$

Ao contrário da TENS de alta frequência e baixa intensidade, que provoca sensação de formigamento não doloroso, a TENS de baixa frequência e alta intensidade ativa aferentes nociceptivos $\mathrm{A} \delta$ e $\mathrm{C}$ de pequeno diâmetro e, portanto, provoca sensações toleráveis, mas dolorosas. ${ }^{25} \mathrm{~A}$ ativação tônica dos aferentes $A \delta$ e $C$ não apenas provoca sensações dolorosas, mas também ativa estruturas do sistema nervoso central, resultando em analgesia. Especificamente, acredita-se que a TENS de baixa frequência e alta intensidade produz analgesia através do recrutamento do sistema de inibição da dor descendente, via a ativação da rede substância cinzenta periaquedural e núcleos da RAFE. ${ }^{25}$

Uma vez que fica claro efeito espacialmente distinto dos dois tipos de TENS, que estes são replicados em populações clínicas, sua aplicação na beira do leito seria imediata. ${ }^{25}$ De fato, a maioria dos estudos clínicos da TENS que investigaram o efeito de diferentes tensões e frequências de estimulação, desconsideraram a possível influência do território do corpo em que a TENS foi aplicada, muitas vezes assumem que os eletrodos da TENS devem ser colocados nas proximidades da área dolorosa. ${ }^{25} \mathrm{~A}$ clara interação entre o local da estimulação e o tipo de TENS indica que grande parte do efeito analgésico máximo da TENS de alta frequência e baixa intensidade é obtido se os eletrodos forem colocados perto da área dolorosa.

Assim, nosso estudo fornece informações importantes para orientar a seleção da melhor combinação de parâmetros de estímulo, intensidade, frequência e localização espacial dos eletrodos, bem como a duração do efeito analgésico produzido pela TENS na prática clínica. Os resultados atuais dão um passo à frente na implementação de tratamentos personalizados para aliviar a dor lombar.

As limitações do nosso estudo incluem o fato do tratamento ofertado ser apenas em uma sessão, o que dificultava o recrutamento dos participantes, consequentemente não foi possível extrapolar o número amostral. A ausência de um grupo controle também não nos permite fazer uma maior inferência deste efeito analgésico.

\section{CONCLUSÃO}

Conclui-se que a TENS apresenta efeitos benéficos imediatos em pacientes com lombalgia. Após as 24 horas, os efeitos ainda se apresentavam, porém, demonstrando um retorno gradual aos níveis da avaliação inicial. Os resultados sugerem que uma nova intervenção dentro do limite de 24 horas deva ser introduzida nesse grupo de pacientes a fim de manter o efeito analgésico alcançado pela TENS. Porém, para confirmação dessa hipótese, novos estudos devem ser conduzidos com mais sessões de TENS para confirmação destes resultados.

\section{AGRADECIMENTO}

Ao Grupo de Estudos e Pesquisas em Reabilitação Esportiva e Traumato Ortopédica da Universidade Luterana do Brasil, Torres/RS, Brasil.

\section{REFERÊNCIAS}

1. Weber KT, Jacobsen TD, Maidhof R, Virojanapa J, Overby C, Bloom O, et al. Developments in intervertebral disc disease research: pathophysiology, mechanobiology, and therapeutics. Curr Rev Musculoskelet Med. 2015;8(1):18-31. Doi: http:// dx.doi.org/10.1007/s12178-014-9253-8

2. Li Y, Fredrickson V, Resnick DK. How should we grade lumbar disc herniation and nerve root compression? A systematic review. Clin Orthop Relat Res. 2015;473(6):1896-902. Doi: http://dx.doi. org/10.1007/s11999-014-3674-y

3. Negrelli WF. Hérnia discal: Procedimentos de tratamento. Acta Ortop Bras. 2001;9(4):39 45. Doi: http://dx.doi.org/10.1590/S141378522001000400005

4. Simon $C B$, Riley JL 3rd, Fillingim RB, Bishop MD, George SZ. Age group comparisons of TENS response among individuals with chronic axial low back pain. J Pain. 2015;16(12):1268-79. Doi: http://dx.doi. org/10.1016/j.jpain.2015.08.009

5. Ebadi S, Ansari NN, Ahadi T, Fallah E, Forogh B. No immediate analgesic effect of diadynamic current in patients with nonspecific low back pain in comparison to TENS. J Bodyw Mov Ther. 2018;22(3):693-9. Doi: http://dx.doi.org/10.1016/j.jbmt.2017.11.003

6. Schulz AP, Chao BC, Gazola F, Pereira GD, Nakanish MK, Kunz RI, et al. Ação da estimulação elétrica nervosa transcutânea sobre o limiar de dor induzido por pressão. Rev Dor. 2011;12(3):231-4. Doi: https:// doi.org/10.1590/S1806-00132011000300007

7. Lima LEA, Lima SO, Rocha CM, Santos GF, Bezerra AJR, Hazime ASO, et al. Estimulação elétrica nervosa transcutânea de alta e baixa frequência na intensidade da dor pós-cesárea. Fisioter Pesq. 2014; 21(3):243-8. Doi: http://dx.doi.org/10.590/18092950/65021032014

8. Morgan CR, Santos FS. Estudo da estimulação elétrica nervosa transcutânea (TENS) nível sensório para efeito de analgesia em pacientes com osteoartrose de joelho. Fisioter Mov. 2011; 24(4):637-46. Doi: https:// doi.org/10.1590/S0103-51502011000400007

9. Ammendolia $C$, Côté $P$, Rampersaud $Y R$, Southerst D, Budgell B, Bombardier C, et al. Effect of TENS versus placebo on walking capacity in patients with lumbar spinal stenosis: a protocol for a randomized controlled trial. J Chiropr Med. 2016;15(3):197-203. Doi: http://dx.doi.org/10.1016/j.jcm.2016.04.001

10. Rajfur J, Pasternok M, Rajfur K, Walewicz K, Fras $B$, Bolach B, et al. Efficacy of selected electrical therapies on chronic low back pain: a comparative clinical pilot study. Med Sci Monit. 2017;23:85-100. Doi: http://dx.doi.org/10.12659/msm.899461

11. Pourahmadi MR, Taghipour M, Ebrahimi Takamjani I, Sanjari MA, Mohseni-Bandpei MA, Keshtkar AA. Motor control exercise for symptomatic lumbar disc herniation: protocol for a systematic review and meta-analysis. BMJ Open. 2016;6(9):e012426. Doi: http://dx.doi.org/10.1136/bmjopen-2016-012426

12. Lins VML, Caldas RR, Carvalho AGC, Barros MFA, Guerino MR, Campos SL, et al. Avaliação da qualidade de vida nos portadores de hérnia de disco lombar praticantes do método pilates. Ter Man. 2013; 11(54):567-72.

13. Malta DB, Oliveira MM, Andrade SSCA, Caiaffa WT, Souza MFM, Bernal RTI. Fatores associados à dor crônica na coluna em adultos no Brasil. Rev Saude Publica. 2017;51 Supl 1:1s-12s. Doi: https://doi. org/10.1590/s1518-8787.2017051000052 
14. Almeida CC, Silva VZMD, Júnior GC, Liebano RE, Durigan JLQ. Transcutaneous electrical nerve stimulation and interferential current demonstrate similar effects in relieving acute and chronic pain: a systematic review with meta-analysis. Braz J Phys Ther. 2018;22(5):347-54. Doi: http://dx.doi.org/10.1016/j. bjpt.2017.12.005

15. Silva MC, Fassa AG, Valle NC. Dor lombar crônica em uma população adulta do Sul do Brasil: prevalência e fatores associados.Cad Saude Publica. 2004;20(2):377-85. Doi: http://dx.doi.org/10.1590/ s0102-311x2004000200005

16. Azevedo JVS, Silva JRL, Ribeiro DCL. Relação entre lombalgia e sobrepeso em praticantes de atividade física. ConScientiae Saúde. 2008;7(4):471-5. Doi: https://doi.org/10.5585/conssaude.v7i4.1384

17. Johnson MI, Paley CA, Howe TE, Sluka KA. Transcutaneous electrical nerve stimulation for acute pain. Cochrane Database Syst Rev. 2015;(6):CD006142. Doi: http://dx.doi.org/10.1002/14651858. CD006142.pub3
18. Tugay $\mathrm{N}$, Akbayrak $\mathrm{T}$, Demirtürk $\mathrm{F}$, Karakaya IC, Kocaacar O, Tugay $U$, et al. Effectiveness of transcutaneous electrical nerve stimulation and interferential current in primary dysmenorrhea. Pain Med. 2007;8(4):295-300. Doi: http://dx.doi. org/10.1111/j.1526-4637.2007.00308.x

19. Dohnert MB, Bauer JP, Pavão TS. Estudo da eficácia da corrente interferencial em comparação à estimulação elétrica transcutânea na redução da dor lombar crônica. Rev Dor. 2015;16(1):27-31. Doi: http://dx.doi.org/10.5935/1806-0013.20150006

20. Ebadi S, Ansari NN, Ahadi T, Fallah E, Forogh B. No immediate analgesic effect of diadynamic current in patients with nonspecific low back pain in comparison to TENS. J Bodyw Mov Ther. 2018;22(3):693-9. Doi: http://dx.doi.org/10.1016/j.jbmt.2017.11.003

21. Mammari MDE, Belfodil AML, Medjahdi MY. Transcutaneous electrical nerve stimulation (TENS) versus physiotherapy for chronic low back pain. Ann Phys Rehabil Med. 2018;61(Supl):e17-e18. Doi: https://doi.org/10.1016/j.rehab.2018.05.038
22. Rebouças Junior FR, Martins LPD, Couto M. Efeito agudo do alongamento por Facilitação Proprioceptiva Neuromuscular associada à TENS e crioterapia na flexibilidade dos músculos isquiossurais de mulheres saudáveis. Fisioter Bras. 2017;18(6):727-33. Doi: http://dx.doi.org/10.33233/fb.v18i6.1094

23. Law PP, Cheing GL, Tsui AY. Does transcutaneous electrical nerve stimulation improve the physical performance of people with knee osteoarthritis? J Clin Rheumatol. 2004;10(6):295-9. Doi: http://dx.doi. org/10.1097/01.rhu.0000147047.77460.b0

24. Bertor WRR, Fracaro GA, Silva LI, Zilio M, Aragão FA, Carvalho AR. Subclassificação da lombalgia crônica e nível de incapacidade: efeito no desempenho funcional e força muscular. Conscientiae Saúde. 2013;12(4):563-71. Doi: https://doi.org/10.5585/ conssaude.v12n4.4514

25. Peng WW, Tang ZY, Zhang FR, Li H, Kong YZ, lannetti GD $\mathrm{Hu}$ L. Neurobiological mechanisms of TENS-induced analgesia. Neuroimage. 2019;195:396-408. Doi: http:// dx.doi.org/10.1016/j.neuroimage.2019.03.077w 